\title{
The Role of Dexa Scan Application in Uremic Patients on Hemodialysis in Ibn-Sina Hemodialysis Center
}

Shakir Hameed Mohamed (FICMS) ${ }^{1}$, Yousif Naser Haded(MbChB, HMD) ${ }^{2}$, Ismail Ibrahim Latif $(\mathrm{MbChB}, \mathrm{PhD}, \mathrm{HMD})^{3}$, Ahmed Methab Athab $(\mathrm{PhD})^{4}$ and Nabeel

Khalid Alwendi (FICMS) ${ }^{5}$

Abstract

Background: Chronic kidney disease is an international public health problem affecting 5$10 \%$ of the world population. As kidney function declines, there is a progressive deterioration in mineral homeostasis, with a disruption of normal serum and tissue concentrations of phosphorus and calcium.

Objective: To determine the effect on bone density by dexa scan and mineral derangement in chronic renal failure, by measuring calicium, phosphate, alkaline phosphatase and parathyroid serum ,total serum protein and Albumin levels and to determine bone density by dexa scan of bones.

Patients and Methods: Cross sectional study was conducted in 75 patients with chronic renal failure. Blood was taken before dialysis session and measurements of serum calcium, phosphorus, serum alkaline phosphatase, .protein and albumin levels, and dexa scan to measure the bone density in these patients.

Results: There was a significant correlation between Hypocalcemia and dexa scan results. Majority of patients had high serum phosphate levels (54.6\%).and normal phosphate level in $40 \%$ while the hypophosphatemia result $5.33 \%$ and mean phosphate level is $2.24 \mathrm{mmol} / \mathrm{l}$. Total serum protein mean is $61.51,37.33 \%$ of them had hypoproteinemia and $62.66 \%$ of them had normal serum protein. There was a significant correlation between dexa scan and vit D level, calcium, alkaline phosphatase, female gender, smoking and body mass index.

Conclusion: The current study demonstrated that it can predict the chronic kidney diseases Metabolic bone disorder from mineral levels before diagnosis by dexa scan.

Keywords: Metabolic bone disorder, dexa scan, chronic kidney disease.

Corresponding Author: ismail_6725@yahoo.com

Received: $10^{\text {th }}$ October 2019

Accepted: $26^{\text {th }}$ November 2019

DOI:https://doi.org/10.26505/DJM.18015011010

${ }^{1,2,5}$ Baquba Teaching Hospital - Diyala-Iraq
${ }^{3,4}$ College of Medicine-University of Diyala-Diyala -Iraq

\section{Introduction}

Chronic kidney disease (CKD) is a world- prevalence [1]. It is associated with a number wide public health problem, with increasing of abnormalities in mineral metabolism such 
as hypocalcemia, hyperphosphatemia and abnormalities in vitamin $\mathrm{D}$ metabolism that result in functional calcitriol deficiency $[2,3,4]$. The major consequences of disordered mineral metabolism in CKD are secondary hyperparathyroidism (SHPT), metabolic bone disease including renal osteodystrophy and vascular calcification $[4,5,6]$. These include parathyroid hormone (PTH), 25-hydroxyvitamin D (25 $(\mathrm{OH}) \mathrm{D}), \quad$ 1,25-dihydroxyvitamin D $(1,25(\mathrm{OH}) 2 \mathrm{D})$, and other vitamin $\mathrm{D}$ metabolites, fibroblast growth factor-23 (FGF-23), and growth hormone. Beginning in CKD stage 3, the ability of the kidneys to appropriately excrete a phosphate load is diminished, leading to hyperphosphatemia, elevated PTH, and decreased 1,25(OH)2D with associated elevations in the levels of FGF-23. The conversion of $25(\mathrm{OH}) \mathrm{D}$ to $1,25(\mathrm{OH}) 2 \mathrm{D}$ is impaired, reducing intestinal calcium absorption and increasing PTH bone loss still remains an important problem due to post hemodialysis factors such as filter size and dialysate fluid type used, persistent HPT, and other metabolic and acid-base disturbances [7].

The therapeutic strategies for alleviation of post HD bone disease include, low dose of calcium, vitamin D, calcimimetic agents, bisphosphonates and, to a lesser extent, calcitonin, good nutrition and avoidance of analgesic and steroid[8].

Despite the knowledge of existence of mineral disorders after hemodialysis, this study was done to determine the severity of bone disease and mineral derangement in chronic renal failure, by measuring calicium, phosphate, alkaline phosphatase and parathyroid serum level,total serum protein and Albumin level and determine dexa scan of bones.

\section{Patients and Methods}

It is a cross-sectional descriptive study.was conducted in Ibn- Sina hemodialysis Center at the Baquba teaching Hospital, Diayala/Iraq. HD patients of 20 years and above who have signed an informed consent. Enrollment was done from September 2016 to April 2017. Stusd sample was 75 of HD patients on regular hemodialysis in ibn-sina dialysis center 51of them were males and 24 females aged between 20 and 78 years were studied and another 25 randomly chosen controlled normal peoples in the same territory was taken to compare results regarding dexa scan results[9].

Patients were those with ESRD on regular HD for 4 hours, three times a week, irrespective to etiology. All dexa scan were done within 2 hours before or after a dialysis session. Inclusion criteria: HD patients who are at least 3 months on HD programme and are on.Follow up at ISC hemodialysis in BTH. Patients who have a bone disorder for other reasons that may affect the results were excluded.

A printed questionnaire was used to collect data from recruited patients. Data consisted of Socio-demographic data including age, gender and dwell area. Information on smoking, alcohol intake, drugs. Medical history for possible etiology of kidney disease, time on dialysis program, since HD 
started and current medications were recorded.

\section{Laboratory Methods}

About $10 \mathrm{ml}$ of blood was drawn before the session of hemodialysis in sterile plain tube for creatinine, urea, calcium, phosphate, total serum protein, vit D3, Alkaline phosphatase, albumin and intact PTH. The measurements of serum creatinine, albumin, calcium and phosphate were determined using automated clinical chemistry analyzer (cobas 400 plus) in IBN-Sina center laboratory, while serum PTH assays were performed in Baquba teaching hospital main laboratory using Enzyme Linked Immuno Sorbent Assay (ELISA) (Cobas e 411).According to the manufacturer instraction. (10-11) the results were analyzed after daily calibration using standard calibration methods and materials and tests assayed against controls. The glomerular filtration rate (GFR) was estimated using the MDRD formula [12] eGFR $=32788$ ラ Serum Creatinine-1.154 x Age-0.203 x [1.212 if Black] $x$ [0.742 if Female] the formula was calculated using a free internet-based clinical equation calculator [13].

Total serum calcium was corrected for serum albumin using the equation: Corrected Calcium $=\mathrm{Ca}$ [40-measured serum albumin] $\mathrm{x}$ $0.02+$ measured serum calcium. (A typical correction is that for every $1 \mathrm{~g} / \mathrm{l}$ that the albumin concentration is below $40 \mathrm{~g} / \mathrm{l}$, the calcium concentration is $0.02 \mathrm{mmol} / \mathrm{l}$ below what it would be if the albumin concentration was normal).All patients sent to Dexa scan unit in Baquba Teaching Hospital were weight,hight ,gender obtained and dexa scan is done for lumber spine and hip area .

\section{Dxa Technology}

A computer with specially designed proprietary software designed by each manufacturer completes the DXA "system."DXA measures bone mineral content (BMC, in grams) and bone area (BA, in square centimeters), then calculates "areal" $\mathrm{BMD}$ in $\mathrm{g} / \mathrm{cm} 2$ by dividing BMC by BA. Tscore, the value used for diagnosis of osteoporosis, is calculated by subtracting the mean BMD of a young-adult reference population from the patient's BMD and dividing by the standard deviation (SD) of young-adult population. Z-score, used to compare the patient's BMD to a population of peers, is calculated by subtracting the mean BMD of an age-, ethnicity-, and sex-matched reference population from the patient's BMD and dividing by the SD of the reference population. The mean $\mathrm{BMD}$ and $\mathrm{SD}$ of the reference populations used for these calculations is a critical variable in the determination of T-scores and Z-scores [20] .

\section{Statistical analysis}

Data analysis was performed using SPSS version 20.0 software. DEXA scan for all patient and controlled were also done and T._score for hip and spine area measured were classified as normal, Osteopenia and osteoporosis. The relationship between bone biochemical parameters and dexa scan results was measured using Pearson correlation coefficient ( $r$ ) and spearman $\mathrm{s}$ correlation coefficient, and linear regression coefficient $(\beta)$. The levels of PTH, calcium and 
The Role of Dexa Scan Application in Uremic Patients on Hemodialysis in Ibn-Sina Hemodialysis Center

Shakir Hameed Mohamed

phosphate were correlated with duration of dialysis, age of the patients was compared between groups using a nova test. All statistical tests were performed at $5 \%$ level of significance ( $95 \%$ confidence interval). The findings were presented using table.

\section{Results}

Table (1): Socio -demographic characteristics of study sample

\begin{tabular}{|c|c|c|c|}
\hline Gender & & BMI & \\
\hline male & $51(68 \%)$ & normal & $23(30.7 \%)$ \\
\hline female & $24(32 \%)$ & overweight & $40(54.7 \%)$ \\
\hline age & & Obese & $11(14.7 \%)$ \\
\hline $20-20$ & $5(6.7 \%)$ & residence & \\
\hline $30-39$ & $11(14.7 \%)$ & Rural & $34(45.3 \%)$ \\
\hline $40-49$ & $12(16 \%)$ & Urban & $41(54.7 \%)$ \\
\hline $50-59$ & $27(36 \%)$ & Socioeconomic state & \\
\hline $60-69$ & $17(22.7 \%)$ & Low & $22(29.3 \%)$ \\
\hline $70-79$ & $3(4 \%)$ & medium & $48(64 \%)$ \\
\hline smoking & $25(33.3 \%)$ & High & $5(6.7 \%)$ \\
\hline alcohol & $6(8 \%)$ & & \\
\hline
\end{tabular}

Most of our patients are male (58\%) with wide distribution of age at presentation but most of them between 50-70 (58\%) and smokers constitute $(33 \%)$ most of them are alcoholic $(8 \%)$. overweight $(54.7 \%)$ possibly fluid overload.

Most of them are medium class regarding socioeconomic state (64\%) and living in urban area $(54.7 \%)$ and small numbers are

Table (2): Patients clinical characteristics

\begin{tabular}{|l||l|}
\hline Duration on dialysis (month) & \\
\hline $4-6$ & $4(5.3 \%)$ \\
\hline \hline $7-12$ & $13(17.3 \%)$ \\
\hline $13-24$ & $20(26.7 \%)$ \\
\hline \hline More than 24 & $38(50,7 \%)$ \\
\hline
\end{tabular}

More than (50\%) are on HD for more than among them. 2 years which indicate good survival rate

Table (3): Patients Medical Status

\begin{tabular}{|l||c||}
\hline \multicolumn{1}{|c|}{ Disease } & present \\
\hline \hline Hypertension & $19(25.3 \%)$ \\
\hline \hline Diabetes & $26(34.7 \%)$ \\
\hline \hline Genitourinary & $15(20 \%)$ \\
\hline \hline Polycystic disease & $3(3.3 \%)$ \\
\hline \hline Unknown cause & $12(16.7 \%)$ \\
\hline
\end{tabular}


The Role of Dexa Scan Application in Uremic Patients on Hemodialysis in Ibn-Sina Hemodialysis Center

Our result showed incidence of uremia in (34.7\%) genitourinary in (20\%) hypertension among patients (25.3\%)as a polycystic disease in(3.3\%) yet we cannot primary cause, diabetes present as cause of define the cause in (16.7\%).

Table (4): Patients history of drug intake

\begin{tabular}{|l||c||}
\hline \multicolumn{1}{|c||}{ Drug history } & present \\
\hline \hline Ferro folic acid & $75(100 \%)$ \\
\hline \hline Calcium carbonate & $70(93 \%)$ \\
\hline \hline Calcitriol(one alfa) & $70(93 \%)$ \\
\hline \hline Sevelamer & $5(7 \%)$ \\
\hline \hline Epirax(erythropoietin) & $75(100 \%)$ \\
\hline
\end{tabular}

Most of our patients are on regular receive erythropoietin supplement and iron treatment of calcium carbonate (93\%) (100\%). calcitriol (one alfa) (93\%) and all of them

Table (5): Biochemical characteristics of the study sample

\begin{tabular}{||l||c||c|c||c||}
\hline & hypo & normal & hyper & mean \\
\hline \hline PTN & $1(1.33 \%)$ & $43(57.33 \%)$ & $31(41.33 \%)$ & $\begin{array}{l}145.62( \pm \\
255.16)\end{array}$ \\
\hline \hline Calicium & $39(52 \%)$ & $35(46.6 \%)$ & $1(1.33 \%)$ & $2.2( \pm 0.2)$ \\
\hline \hline Phosphate & $4(5 \%)$ & $30(40 \%)$ & $41(55 \%)$ & 2.4 \\
\hline \hline $\begin{array}{l}\text { Alk. } \\
\text { Phosphatase }\end{array}$ & $2(2.6 \%)$ & $50(66.6 \%)$ & $23(30.6 \%)$ & 123.23 \\
\hline \hline Vit D & $73(97.3 \%)$ & $2(2.66 \%)$ & & 38.3 \\
\hline \hline $\begin{array}{l}\text { Total serum } \\
\text { protien }\end{array}$ & $47(62.66 \%)$ & $28(37 \%)$ & & 61.51 \\
\hline \hline $\begin{array}{l}\text { Serum } \\
\text { albumen }\end{array}$ & $56(74.66 \%)$ & $19(25.33)$ & & 30.11 \\
\hline \hline
\end{tabular}

Most of our patients have normal PTH $(57.33 \%)$ and increase level of PTH in $(41.33 \%)$.regarding calcium level majority have low calcium $(52 \%)$ and normal calcium level in 46.6.phosphate are high in (55\%) and normal in (40\%).alk.phosph are normal in (66.6\%) but elevated level present in only (30.6\%).VIT D are low in almost all patients (97.3\%).most of patients have low TSP and low Albumen $(62.66 \%)(74.66 \%)$ respectively.

Table (6): Dexa scan results in study Sample

\begin{tabular}{|l||c||c||c||}
\hline & Normal & Osteopenia & osteoporosis \\
\hline \hline Dexa scan total & $20(26.66 \%)$ & $35(46.66 \%)$ & $20(26.66 \%)$ \\
\hline \hline Dexa scan hip & $27(32 \%)$ & $37(49.33 \%)$ & $11(14.66 \%)$ \\
\hline \hline Dexa scan spine & $33(44 \%)$ & $25(33.33 \%)$ & $17(22.66 \%)$ \\
\hline
\end{tabular}


Dexa scan showed that nearly half $(46.66 \%)$ of patients have osteopenia and only quarter have osteoporosis (26.66\%). There is very significant anova regression between mineral (biomarkers) and dexa scan result for future relation and dependable.

\section{Discussion}

The chronic kidney disease is a worldwide public health problem associated with high morbidity and mortality (14-15).Secondary hyperparathyroidism after HD has been reported even in the presence of excellent HD [16].

In this study, CKD on HD programme, calcium, phosphorus and PTH considerably normalized in majority of the patients. The mean ages of our patients $54.5 \mathrm{yrs}$, the common age groups affected 50-59 y are $27(36 \%)$, then age group 60-69 y were $17(22.7 \%)$.there is significant correlation between age and hyper tension $(p=.040)$ and with $\operatorname{BMI}(\mathrm{p}=.000)$.

Our study is agree by a study done by Evenepoel et al and Kawarazaki et al 54.5 and 50.0 years respectively [17]. A study done by Sidney Klawansky, et al., showed that there are links between osteoporosis increased mortality, possibly reflecting links of low BMD with poor nutritional status and vascular calcification[19].In our study, males made up the majority of the patients with51 (68\%) and females 24(32\%)this goes with other study done in Africa (Nigeria, Senegal and Burkina Faso) have also reported a male preponderance among patients with ESRD [19]. In one study by $M$ Asaka, H Iida, M FUJITA, K IZUMINO, M
TAKATA, H SETO, and S SASAYAMA, female was inversely correlated with BMD with age, but not in male HD patients [20]. Which may be due to hormonal changes and exercise reduction with chronic diseases. Female gender has also been linked as an independent risk factor. This was negatively related with BMD, suggesting that the female skeleton is more vulnerable CKD_MBD changes than that of the male [21]. Regular weight-bearing, physical activity also had a beneficial effect on bone mass, while prolonged bed rest had a powerful negative impact on skeletal health [22].This may explain difference between genders in BMD. The majority of patients in our study were overweight 41(54.6\%), and 11 (14.6\%) obese but only $23(30.66 \%)$ normal. This possibly because many patients have fluid overload and didn't reach the dry weight The higher level of PTH mean was with overweight group also major reduction of vit D occur in over weight group and lowest in normal weight group for both 41.84(-+13.93), 2.01(+1.188 ) respectively. There is no significant Anova correlation between BMI with PTH,Ca,po4 and age but high significant with alkaline phosphatase and also significant person s correlation between BMI and sex which are similar to other studies results. There was a statistically significant relationship between increased age of the patients as well as duration of dialysis and a high PTH level $(\mathrm{p}<0.001)$ [18]. The study by Bess Dawson-Hughes et al., there is a positive correlation between body weight and BMD in the general population [23]. 
Similarly, several studies demonstrated that the body size relates with BMD also in CKD and ESRD patients $[24 ; 25 ; 26]$.A study done in Turkey among renal HD, low BMI values and decreased $25 \mathrm{OH}$ vitamin $\mathrm{D}$ levels were found to be a main risk factors for loss of bone mineral density[27]. Priyanka et al reported a positive correlation between BMI and bone mineral density in spine and femur. Longer CKD duration prior to HD showed greater loss of BMD in the femur[28]. In general body mass index associates with BMD or bone mass as measured by DEXA and the BMI is a predictor of BMD also in ESRD patients [29]. Thus, not surprisingly, body weight and BMI were primary responsible factors for BMD variation, osteoporosis and fractures (30.31). Almost half $39(52 \%)$ of our study population had hypocalcemia, we found no significant correlation between age group nor duration of dialysis with hypocalcemia.

We found significant correlation between hypocalcemia and estimated T_score and no with BMI. Only 1(1.33\%) of our patients had hypercalcemia as it has been reported in other studies with hypercalcemia ranging from $3 \%-21 \%[18,33,34,35,36]$. In the early post HD period serum calcium has been reported to follow a biphasic pattern with an immediate post HD decline followed by a significant increase. All 100\% patients were on calcium supplements regularly despite having different calcium level because our patient compliance is less than other population and a bad minded about hemodialysis programme and poor patients and nutritional and gastrointestinal complication of uremia. In this study vitamin D levels were low determined and thus a correlation with low serum calcium levels in this population could be ascertained $(37,11)$. In my study, nearly as half as $41(54.6 \%)$ of renal HD patients had high serum phosphate Kawarazaki et al., reported hypophosphatemia among 15\% of HD 12 month duration. Hypophosphatemia was associated with persistent HPT 12 months of HD The difference in the prevalence of hypophosphatemia might be due to the fact that in the study by Kawarazaki et al the mean PTH levels at 12 months duration of HD was higher 107 (71.0-205.3)pg./ml compared to a mean of 2.24(-+1.44) (25.058.6)pg./ml at a median duration of (13-24) months HD in this study. The mean of serum phosphate in the early three months of dialysis was $2.300 \mathrm{mmol} / \mathrm{l}$.

Serum phosphorus levels are usually elevated prior to renal dialysis and decreases rapidly following $\mathrm{HD}$ to within or below range for patients with normal kidney function in the first few post HD months $(38,39)$. The results in my study were $73(97.33 \%)$ have vit D deficiency and $2(2.33 \%)$ of patient were normal. This study results are go with that done by Grahame J Elder (40) but not agree by study in japan by K Nakatsuka and Y Nishizawa. Vitamin D supplementation reduces serum PTH levels and improves bone strength. The results of our study, the dexa scan as a whole show 20 $(26.66 \%)$ of patients with normal BMD, and $35(46.6 \%)$ with osteopenia BMD, and 
$20(26.66 \%)$ of them having osteoporotic BMD and no fractures in between but there is considerable difference in BMD between the hip area t-score and spine area t-score. Shows that $27(32 \%)$ were normal BMD $37(49.33 \%)$ had osteopenia and 11(14.66\%) of them with osteoporosis. The osteopenia of hip associated with lower values of PTH, PO4, Ca while osteoporotic hip associated with highest elevation of PTH, ALP and po4 but with lowest most values of Ca, VIT D, total serum protein and albumin. There is significant correlation in anova table between hip t-score and PTH $(\mathrm{p}=.000)$ and with serum albumin $(\mathrm{p}=.000)$ while no significant correlation with PTH in Duncan test nor with albumin level. In spearman s rho there is very significant correlation between hip T-score and serum albumin $(\mathrm{p}=.000)$ and with alkaline phosphatase $(\mathrm{p}=.004)$ and also with total serum protein $(\mathrm{p}=.000)$ and no significant correlation with $\mathrm{Ca}$ or po4.

\section{Conclusions}

1-This study demonstrated that elevated serum PTH levels are present even after HD and are associated with many factors for bone diseases. It also showed presence of low PTH levels though the prevalence is lower than those reported in the CKD counterpart. This may indicate continuing presence of a dynamic bone disease if PTH levels are used as surrogate markers for bone turnover.

2-Hypocalcemia was present in almost half of HD as opposed to hypercalcemia reported in other studies. This may be as a result of higher prevalence of a dynamic bone disease in our CKD patients. Hypocalcemia was associated with relatively older patients and was not correlated with duration of dialysis. 3-Nearly half of our patients had serum phosphorus levels within reference ranges.

4-There is great predictable value can be obtained from mineral analysis for patient with mineral and bone diseases and used as a good indicator for follow up of them without need to do dexa scan and can be depended on these biochemical markers as screening tests to evaluate the bone mineral diseases especially when dexa scan is not available in some peripheral hospitals or areas.

\section{References}

[1]Pendse S, Singh AK. Complications of chronic kidney disease: Anemia, mineral metabolism, and cardiovascular disease. Med Clin North Am. 2005;89:549-61. [PubMed] [2]Martin KJ, Olgaard K, Coburn JW, Coen GM, Fukagawa M, Langman C, et al. Diagnosis, assessment, and treatment of bone turnover abnormalities in renal osteodystrophy. Am J Kidney Dis. 2004;43:558-65.[PubMed]

[3]Kronenberg F, Mündle M, Längle $M$, Neyer U. Prevalence and progression of peripheral arterial calcifications in patients with ESRD. Am J Kidney Dis. 2003;41:1408. [PubMed]

[4]Martola L, Barany P, Stenvinkel P. Why do dialysis patients develop a heart of stone and bone of china? Blood Purif. 2005;23:203-10. [PubMed]

[5]Raggi P, Boulay A, Chasan-Taber S, Amin N, Dillon M, Burke SK, et al. Cardiac calcification in adult hemodialysis patients. A link between end-stage renal disease and 
cardiovascular disease? J Am Coll Cardiol. 2002;39:695-701. [PubMed]

[6]Norris KC, Crooks PW, Nebeker HG, Hercz G, Milliner DS, Gerszi K, et al. Clinical and laboratory features of aluminum-related bone disease: Differences between sporadic and "epidemic" forms of the syndrome. Am J Kidney Dis. 1985;6:342-7. [PubMed]

[7]Dawn Shao TL, Terence Yi SK, Stephenie FC et al. Prevalence and Patterns of Bone Loss in the First Year After Renal Transplant in South East Asia Patients. Transplantation 2011;92:557-563.

[8]Jose RW, Raul GC, Eudocia R et al. Bone disease after transplantation. Clin J Am Soc Nephrol 2006; 1: 1300-1313.

[9]Daniel, W. W. Biostatistics: A Foundation for Analysis in Health Sciences. 7th edition. New York: John Wiley \& Sons. 1999. [10]Stavroulopoulos A, Cassidy M J D, Porter CJ et al. Vitamin D Status in Renal Transplant Recipients. Am J Transplant 2007; 7: 2546-2552.

[11]Taziki O, Espahbodi F, Alizadeh Forutan $\mathrm{M}$ et al. 25-Hydroxyvitamin D Deficiency in Kidney Transplant Recipient. Iran J Kidney 2011; 5 (1): 57-62 .

[12]Levey AS, Bosch JP, Lewis JB et al. A more accurate method to estimate glomerular filtration rate from serum creatinine: a new prediction equation. Modification of Diet in Renal Disease Study Group. Ann Intern Med 1999; 130 (6): 461-70.

[13] Dousdampanis P, Trigka K, Fourtounas $\mathrm{C}$ et al. Evolution of secondary hyperparathyroidism one year after successful renal transplantation. Hippokratia 2011.15(2): 30-32.

[14]Grotz WH,Mundinger FA, Goged B et al.Bone mineral density after kidney transplantation 1995;59:982-986.

[15]Rojas E, Carlini RG, Clesca P et al. the pathogenesis of osteodystrophy after Renal Transplantation as Detected by Early Alterations in bone Remodelling.Kidney Int 2003;63:1015-1023.

[16]Saha HH ,Salmela KT,Ahonen PJ.et al,Sequential changes in vitamin $\mathrm{D}$ and calcium metabolism after successful renal transplantation. Scand J Urol nephrol 1994;28(1):21-27.

[17]Kawarazaki H,Shibagaki Y, Fukumoto S et al,Natural history of mineral and bone disorders after living-donor kidney transplantation. A one year prospective observational study. Therapeutic apharesis and dialysis 2011;15(5):481-487.

[18]Gholamhossein RO, Mohamad HD, mohammadS et al.predictive factors for persistent hyperparathyroidism after kidney transplantation.Arch Iranian Med 2005;8(4):295-299.

[19]Sidney Klawansky, Eugene Kom Paul F Cavanaugh Jr, David Y.relationship age ,renal function and bone mineral density in the US population Osteoporosis international,14(7):570\{576,2003\}

[20]Naicker S. End-stage renal disease in Sub- Saharan and South Africa. Kidney Int Suppl. 2003;83: S119-22.

[21] M Asaka, $\mathrm{H}$ Iida, $\mathrm{M}$ FUJITA, $\mathrm{K}$ IZUMINO, M TAKATA, H SETO, and S SASAYAMA. Total and regional bone 
mineral density by dual photon absorptiometry in patients on maintenance hemodialysis. Clinical nephrology, 38(3):149\{153, 1992\}.

[22]Ugur A, Guvener N, Isklar I et al. Osteoporosis after Renal Transplantation: Single Center Experience. Transplantation 2001;71:645-649.

[23]Marcel J, Cambe B, Francisco J et al. Bone Mineral Density of 704 Amateur Sportsman Involved in Different Physical Activities. Osteoporos Int 2001;12:152-157. [24]Bess Dawson-Hughes, Clanton Shipp, Laura Sadowski, and Gerard Dallal. Bone density of the radius, spine, and hip in relation to percent of ideal body weight in postmenopausal women. Calcied tissue international, 40(6):310\{314, 1987.

[25]Abderrahmane Ghazali, Franck Grados, Roxana Oprisiu, Delia Bunea, Philippe Morini_x0012_ere, Najeh El Esper, Isabelle El Esper, Michel Brazier, Jean Claude Souberbielle,Albert Fournier, et al. Bone mineral density directly correlates with elevated serum leptin in haemodialysis patients. Nephrology Dialysis Transplantation, 18(9):1882\{1890, 2003\}.

[26] Maarten W Taal, Tahir Masud, Desmond Green, and Michael JD Cassidy. Risk factors for reduced bone density in haemodialysis patients. Nephrology Dialysis Transplantation, 14(8):1922\{1928, 1999.

[27]Fettah Fevzi Ersoy, Stauros Ploumis Passadakis, Paul Tam, Evaggelos Dimitros Memmos, Pericles Konstantinos Katopodis, $\mathrm{C}$ etin $\square$ Ozener, Fehmi Akcicek, Taner C amsar_x0010_, Kenan Ates, Rezzan Ataman, et al. Bone mineral density and its correlation with clinical and laboratory factors in chronic peritoneal dialysis patients. Journal of bone and mineral metabolism, 24(1):79\{86,2006\}.

[28]Unal A, Kocyigit L, Sipahioglu MH et al. Loss of Bone Mineral Density in Renal Transplantation Recipients. Transplant Proc. 2010; 42(9):3550-3.

[29]Priyanka G, George A, Balaji P et al. Bone Mineral Disease in Renal Transplantation- An Indian Experience. JNRT 2009; 2(1): 63-70.

[30]Marianne Relle Andreassen, Peter Eskildsen, Bente Langdahl, and KlausOlgaard. Bone mineral density and biochemical markers of bone turnover in patients with predialysis chronic renal failure. Kidney international, 56(3):1084\{ 1093, 1999.

[31]Catherine O Stehman-Breen, Donald J Sherrard, Astier M Alem, Daniel L Gillen, Susan R Heckbert, Craig S Wong, Adrianne Ball, and Noel S Weiss. Risk factorsfor hip fracture among patients with end-stage renal disease. Kidney interna- tional, 58(5):2200\{2205, 2000.

[32] Shu-Feng Lei, Fei-Yan Deng, Miao-Xin Li, Volodymyr Dvornyk, and Hong-Wen Deng. Bone mineral density in elderly chinese: e ects of age, sex, weight, height and body mass index. Journal of bone and mineral metabolism, 22(1):71\{78,2004\}.

[33]Csaba A. Bone mineral disorders in patients on maintainance hemodialysis and after kidney transplantation: MD Thesis, 2009. Semmelweis University. Budapest. 
[34]Pieter E, Kathleen C, Dirk K et al. Natural History of Parathyroid Function and Calcium Metabolism after Kidney Transplantation. Nephrol Dial Transplant 2004;19:1281-1287.

[35]El Maghraoui, Borderie et al. 1999; Maillefert, Aho et al. 2001; El Maghraoui 2004; El Maghraoui 2004; El Maghraoui, Do Santos Zounon et al. 2005.

[36]Pieter E, Barbara V, Maarten N et al. Calcium Metabolism in the Early Post Transplantation Period. Clin J Am Soc Nephrol 2009; 4(3): 665-672.

[37]Hamidian Jahromi A, Roozbeh J, RaissJalali GA, et al. Risk Factors of Post Renal Transplant Hyperparathyroidism. Saudi J Kidney Dis Transpl 2009; 20(4): 573-576.

[38]Stavroulopoulos A, Cassidy M J D, Porter CJ et al. Vitamin D Status in Renal Transplant Recipients. Am J Transplant 2007; 7: 2546-2552.

[39]El-Amm JM, Doshi MD, Singh A, et al. Preliminary experience with cinacalcet use in persistent secondary hyperparathyroidism after kidney transplantation. Transplantation 2007; 83(5):546-9.

[40]Kamar N, Gennero I, Spataru L, et al. Pharmacodynamic effects of cinacalcet after kidney transplantation: once-versus twicedaily dose. Nephrology Dialysis Transplantation 2008; 23:3720-4.

[41]Grahame J Elder Vitamin d levels, bone turnover and bone mineral density show seasonal variation in patients with chronic kidney disease stage 5 Nephrology, 12(1):90\{94, 2007\}. 